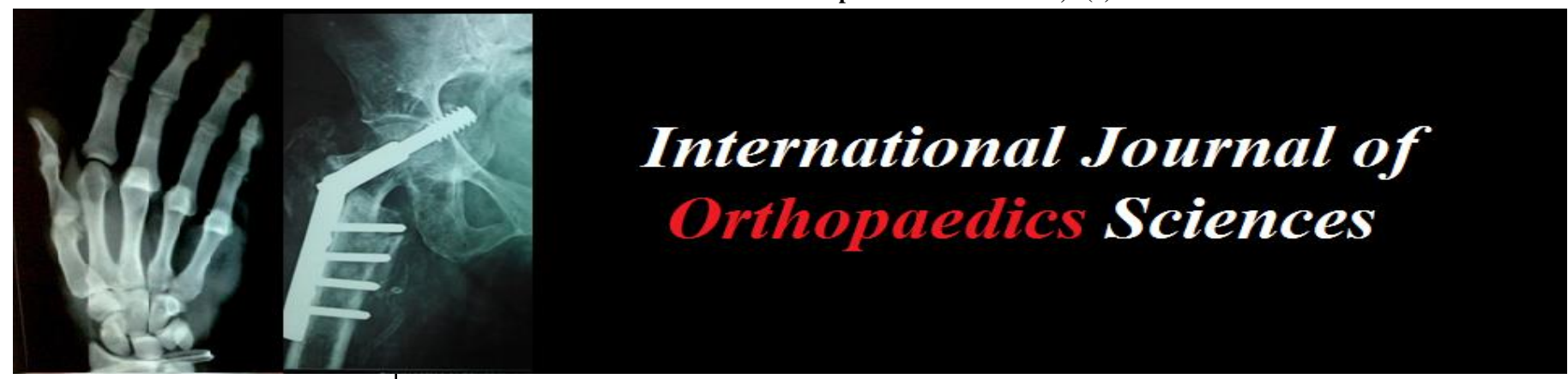

E-ISSN: 2395-1958

P-ISSN: 2706-6630

IJOS 2021; 7(2): 455-460

(C) 2021 IJOS

www.orthopaper.com

Received: 25-02-2021

Accepted: 27-03-2021

Dr. Akshay Nayak

M.S. Orthopaedics, Bonded

Assistant Professor, Lokmanya

Tilak Municipal Medical College

and General Hospital, Sion,

Mumbai, Maharashtra, India

Dr. Arvind B Goregaonkar

M.S. Orthopaedics, Head of the

Department, Department of

Orthopaedics, Lokmanya Tilak

Municipal Medical College and

General Hospital, Sion, Mumbai,

Maharashtra, India

Dr. Vishal Raina

M.S. Orthopaedics, Bonded

Assistant Professor, Department

of Orthopaedics Lokmanya Tilak

Municipal Medical College and

General Hospital, Sion, Mumbai,

Maharashtra, India

Dr. Nirav R Gupta

M.S. Orthopaedics, Bonded

Assistant Professor, Lokmanya

Tilak Municipal Medical College and General Hospital, Sion,

Mumbai, Maharashtra, India

Dr. Shreenidhi K Kulkarni

M.S. Orthopaedics, Bonded

Assistant Professor, Lokmanya

Tilak Municipal Medical College and General Hospital, Sion,

Mumbai, Maharashtra, India

Corresponding Author: Dr. Akshay Nayak

M.S. Orthopaedics, Bonded Assistant Professor, Lokmanya Tilak Municipal Medical College and General Hospital, Sion,

Mumbai, Maharashtra, India

\section{Prospective study of short term functional outcome in elderly patients treated with cemented vs uncemented bipolar hemiarthroplasty for transcervical neck of femur fractures}

\author{
Dr. Akshay Nayak, Dr. Arvind B Goregaonkar, Dr. Vishal Raina, \\ Dr. Nirav R Gupta and Dr. Shreenidhi K Kulkarni
}

DOI: $\underline{\text { https://doi.org/10.22271/ortho.2021.v7.i2f.2664 }}$

Abstract

Objective: Transcervical femur neck fractures are common occurrences in elderly age group leading to significant impact in the health care setup. Bipolar hemiarthroplasty is the commonly performed surgery in this age group. There has been an ongoing debate based on outcome of cemented vs uncemented hemiarthroplasty. The purpose of the study is to evaluate the functional outcome with individual techniques assessed by the Harris Hip Score.

Materials and Methods: It was a single centre prospective study with the total sample size of 50 . Randomisation was done into 2 groups, one to be treated with uncemented bipolar hemiarthroplasty and the other one with cemented hemiarthroplasty. The comparison was done based on the demographic data, intraoperative parameters, post-operative complications and functions. The patients were followed up till one year and functional outcome was evaluated using the Harris Hip Score.

Results: The average age of the patients in the study is 75.02 years , with most patients between $70-80$ years and females $(54 \%)$ being more than males $(46 \%)$. Surgical time and blood loss was greater for the cemented $(94.4 \mathrm{~min}$ and $234.8 \mathrm{ml})$ cohort than the uncemented $(60.80 \mathrm{~min}$ surgical time and $183.20 \mathrm{ml}$ blood loss) ( $p$ value $<0.001$ ). However the functional outcome at the end of one year was the similar in both the study groups. Mean Harris Hip Score at 1 year for cemented group was 94.1 and uncemented group was 94.43 with $\mathrm{p}$ value $=0.862$ (not significant).

Conclusion: Based on our results the advantages of using an uncemented hemiarthroplasty are less intraoperative bleeding, shorter duration of surgery which was statistically significant. Both cemented and uncemented hemiarthroplasty are accepted modality of treatment in transcervical femur neck fractures in elderly, as there is no statistical difference in the functional outcome at the end of one year.

Keywords: transcervical neck fractures, cemented hemiarthroplasty, uncemented hemiarthroplasty, harris hip score

\section{Introduction}

Femoral neck fractures are a rising problem in our aging society, frequently troubled with multiple and severe co-morbidities, and are associated with high morbidity and mortality. That with disability and mortality imposes high health care costs on the health system. Epidemiologic studies have recognized several risk factors for femoral neck fracture, including $\mathrm{BMI}<18.5$, Insufficient sunlight, low activity, smoking, history of osteoporosis related fracture, positive history of hip fracture in his or her mother and treatment with corticosteroid. The usual cause of this fracture is a simple fall in which force is transmitted from greater trochanter to femoral neck ${ }^{[1]}$. Other mechanism is leg external rotation with increased force on the capsule and iliofemoral ligament. Intracapsular femoral neck fractures account for about $50 \%$ of hip fractures. The union rate is low because of low blood supply and intracapsular situation; it is also sometimes associated with femoral head necrosis and delayed segmental necrosis. In recent years, the improvement of health services and increased life expectancy has dramatically increased the incidence of this type of fracture. 
Hemiarthroplasty (HA) using modular head partial prostheses is a common surgical procedure used to treat elderly patients with femoral neck fractures. Hip hemiarthroplasty is superior to internal fixation for displaced femoral neck fractures, enabling earlier mobility, less reoperations, and better functional outcome at one year. However, controversy persists as to whether cemented or uncemented HA is preferable for elderly patients. The discussion about cemented or uncemented HA is similar to the discussion about cemented or uncemented prostheses in primary total hip arthroplasty.

There are different types of cement and uncemented bipolar prosthesis. This prosthesis has an articular surface between the head and shell and articular surface between the acetabulum and shell. Tow joint prosthesis are likely to reduce wear and protrusion to the acetabulum. We can use orthopedic cement for stability of stem into femoral canal to increase the stability of stem and decrease loosening rates; in contrast, this can lead to complications such as increased intraoperative bleeding and embolism.

The purpose of this prospective study is to compare a cemented and uncemented hemiarthroplasty. Considering good number of fracture neck femur encountered in our hospital, I intend to do this clinical study and results will be evaluated in comparison with Harris hip score.

\section{Materials and Methods}

Design: Single centre, Prospective study.

Place of study: Tertiary care centre in the city of Mumbai Sample size: 25 patients with cemented hemiarthroplasty and 25 uncemented hemiarthroplasty

Sampling method: In this study, all the patients presenting with traumatic transcervical neck of fractures in elderly are included. Randomisation was done into 2 groups, one to be treated with uncemented bipolar hemiarthroplasty and the other one with cemented hemiarthroplasty .

\section{Inclusion Criteria}

1. Any individual above 60 years of age with traumatic transcervical femur neck fracture.

\section{Exclusion criteria}

1. Any individual below 59 years of age.

2. Pathological fractures.

3. Intertrochanteric femur fractures.

4. Pre-existing hip arthritis.

5. Type C Dorr canal

After approval of the ethics committee, this observational study was conducted. It involved patients posted for surgery after satisfying the inclusion and exclusion criteria. Written valid informed consent was taken from the respective patients. At the time of admission detailed physical examination was done. Imaging studies was done i.e anteroposterior view of pelvis with both hips and lateral films of the affected hip to diagnose the fracture. Demographic data, detailed history of symptoms and other co-morbidities was be recorded. Skin traction was applied to all patients in the ward. Routine biochemical and haematological investigations was assessed.

After randomisation, the patients posted for surgery. Hip was approached posteriorly following the Southern Moore approach. Intraoperative assessment was done based on the amount of blood loss, duration of surgery, any incidence of cement reaction, periprosthetic fractures or any conversion of uncemented to cemented hemiarthroplasty.

Intravenous post-operative antibiotics was given for 3 days. Analgesics was given as per the patients compliance Limb length discrepancy was noted. VAS (Visual Analogue Scale) score of the patient noted for all post-operative patients. Patients were made to sit up on the second day, encouraged to perform static and dynamic quadriceps exercise, stand-up with support (walker) on the first or second day (full weight bearing for the cemented group and partial weight bearing for the uncemented group) and were allowed walk with the help of a walker depending on his/her pain tolerance and were encouraged to walk thereafter. Post-operative radiograph was taken as antero-posterior view of pelvis with both hips. Drain removal was done after 48 hours. Sitting cross-legged and squatting were not allowed. Suture removal was done on the fifteenth postoperative day. Patients were asked to come for follow up after one month, 3 months, sixth month and one year. At follow up, detailed clinical examination was done systematically. Infection was ruled out. Patients were evaluated according to Harris hip scoring system for pain, limp, the use of support, walking distance, ability to climb stairs, ability to put on shoes and socks ( in our study for some patients ability to wash feet was enquired ) sitting on chair, ability to enter public transportation, deformities, leg length discrepancy and movements. All the details were recorded in the follow up chart. The radiograph of the operated hip was taken at regular intervals, at each follow up. X-Rays were taken to rule out any loosening, subsidence, periprosthetic fractures.

Total Functional outcome was graded at each follow up based on Harris Hip Score.

Poor - Harris Hip Score $<70$

Fair - Harris Hip Score 70-80

Good - Harris Hip Score 80-90

Excellent - Harris Hip Score $>90$ 


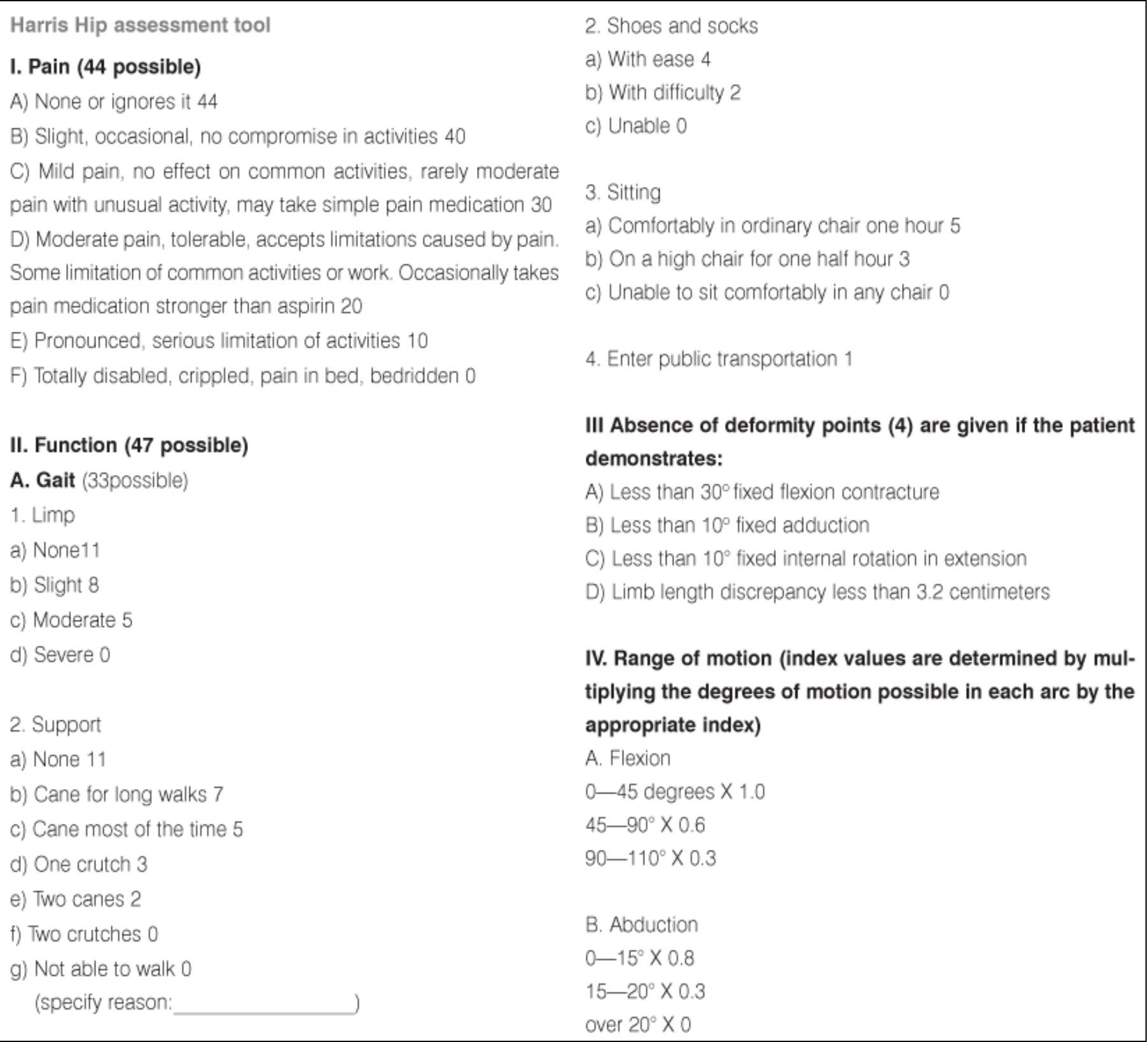

Fig 1: Harris Hip Assessment Tool

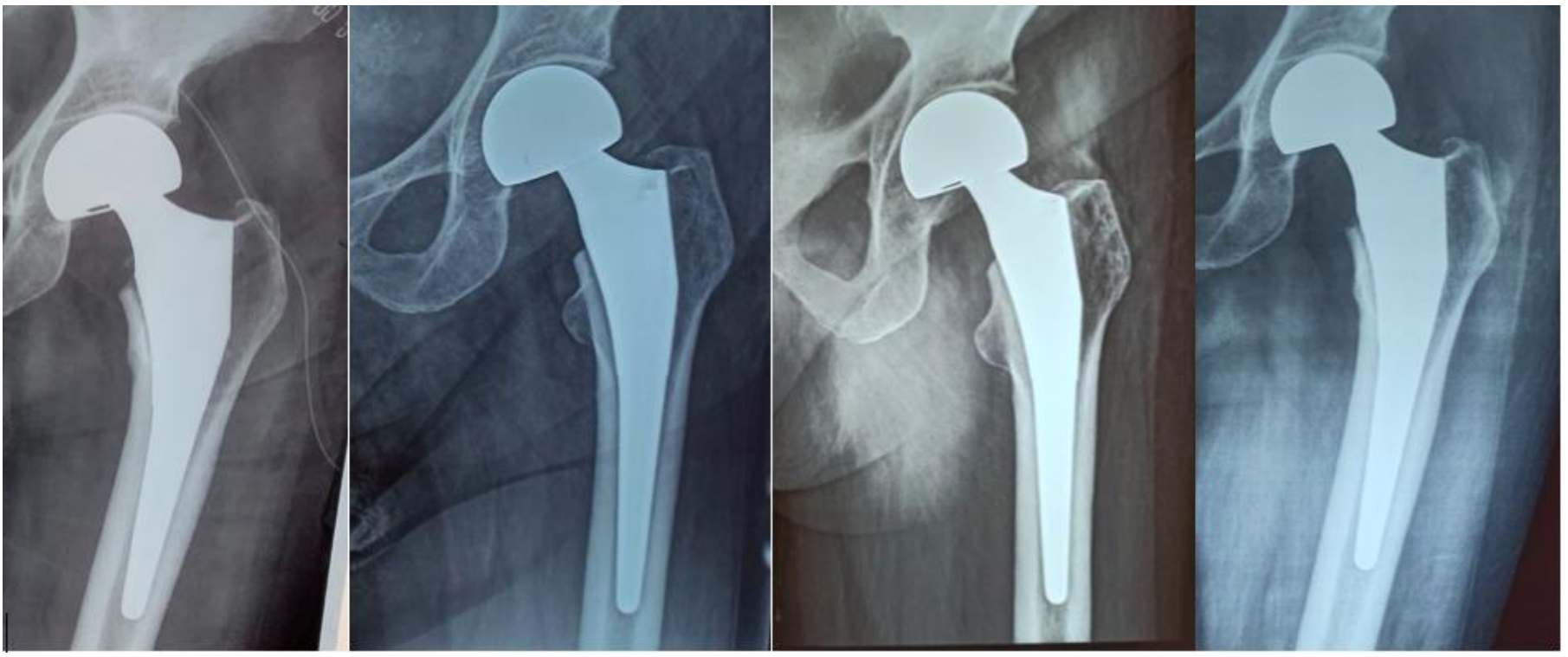

Fig 2: Post-operative and follow up (1, 3, 12 months) radiographs of a case treated with uncemented hemiarthroplasty 

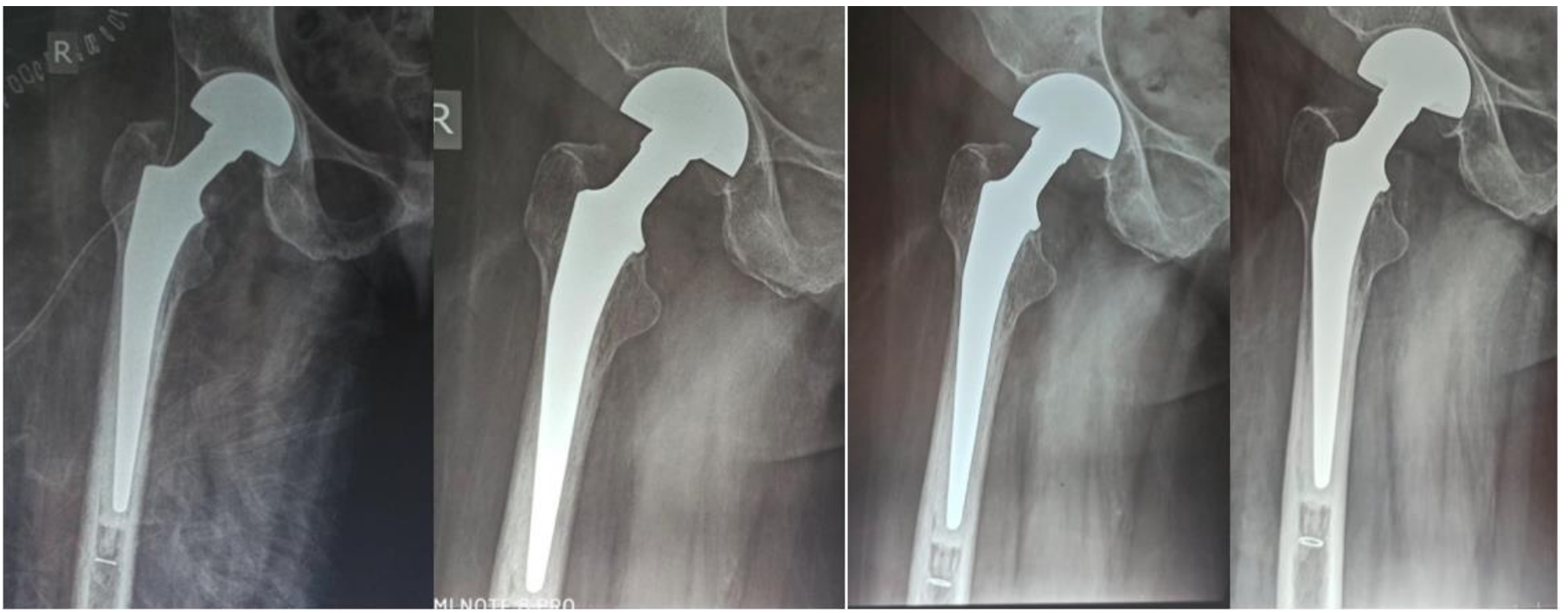

Fig 3: Post-operative and follow up (1, 3, 12 months) radiographs of a case treated with cemented hemiarthroplasty

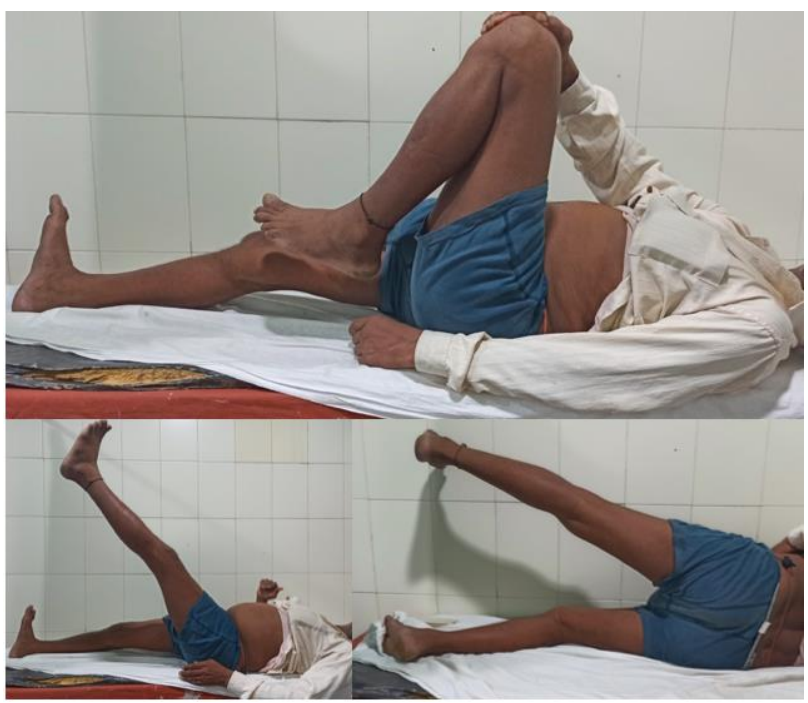

Fig 4: Picture showing functional outcome at one year follow up

\section{Results}

The average age of the patients in the study is 75.02 years, with most patients between $70-80$ years. Maximum age was 87years and minimum age of 64 years. The Mean age in cemented group was 75.56 years (SD-4.86). The Mean age in uncemented group was 74.48 years (SD-7.73). Total number of females in the study were $27(54 \%)$ and number of male were $23(46 \%)$. Out of 50 patients, 27 patients had trauma to right side and 23 had trauma to the left side. Majority of the patients had trauma due to fall at home due to slip in the bathroom or while walking (84\%). 16 percent of patients had history of road traffic accident. On an average patients were operated after 5.8 days after trauma in both the groups.

In the cemented group the mean duration of surgery was 94.40 minutes with a mean amount of blood loss of 235.80 millilitres, whereas in the uncemented group the mean duration of surgery was 60.80 minutes and mean amount of blood loss being 183.20 millilitres . Surgical time and blood loss was greater for the cemented cohort than the uncemented. The difference between the groups was significant $(\mathrm{P}=$ 0.0001). All the cases were stable intraoperatively. There were no cases of intraoperative dislocation or peri prosthetic fracture. there were no conversion of uncemented bipolar hemiarthroplasty to cemented ones.

Table 1: Comparison of Operative Time and Blood Loss between Cemented and Uncemented Groups (N=50)

\begin{tabular}{|c|c|c|c|}
\hline \multirow{2}{*}{ Parameter } & \multicolumn{2}{|c|}{ Group } & \multirow{2}{*}{ P Value } \\
\cline { 2 - 4 } & Cemented (n=25) n (\%) & Uncemented (n=25) n (\%) & \\
\hline Operative Time (min) & $94.40(12.18)$ & $60.80(11.42)$ & $<0.001^{*}$ \\
\hline Blood Loss (ml) & $235.80(37.46)$ & $183.20(29.11)$ & $<0.001^{*}$ \\
\hline \multicolumn{2}{|c|}{ Unpaired t Test, P Value *Significant } \\
\hline
\end{tabular}

In the cemented group, 24 percent of patients had VAS (Visual Analogue Scale) score of 4,64 per cent had VAS 5, and 12 percent had VAS 6 . In the uncemented group, 44 percent had VAS 4, 44 percent had VAS 5, and 12 percent of patient had VAS 6.

Superficial infection was seen in 3 cases $(12 \%)$ of cemented bipolar hemiarthroplasty and 2 cases $(8 \%)$ of uncemented hemiarthroplasty which was treated with surgical debridement and intravenous antibiotics, followed by oral antibiotics. There was one case of deep infection in cemented group who expired during the course of treatment. There were total 4 cases $(16 \%)$ of mortality in cemented group and 2 cases $(8 \%)$ in uncemented group. There were no cases of bone cement implantation syndrome. There were no cases of postoperative periprosthetic fractures, dislocations, deep vein thrombosis, loosening or subsidence.

The Mean Harris Hip Score at first month for cemented group (73.68) was slightly better (although insignificant as p value < 0.05) than uncemented group (71.28), due to the early full weight mobilization for the cemented group. The Mean Harris Hip Score of cemented group was 83.09 (good) and uncemented group was 86.88 (good) (Unpaired t Test, P Value $=0.365$, Not Significant). The Mean Harris Hip Score of cemented group was 91.75 (excellent) and uncemented group was 93.17 (excellent) (Unpaired $\mathrm{t}$ Test, $\mathrm{P}$ Value = 0.521 , Not Significant)

However at the end of one year the mean Harris Hip Score of cemented (94.1) and uncemented group (94.43) was similar (P 
Value $=0.862$, Not Significant). 15 patients $(71.43 \%)$ in the cemented group had excellent outcome and 6 patients $(28.57 \%)$ had good outcome. 16 patients (69.57) in the uncemented group had excellent outcome and 7 (30.43\%) patients had good outcome.

Table 2: Comparison of Mean Harris Hip Score between Cemented and Uncemented Groups at different time intervals $(\mathrm{N}=50)$

\begin{tabular}{|c|c|c|}
\hline Interval & $\begin{array}{c}\text { Mean Harris hip } \\
\text { score (cemented) }\end{array}$ & $\begin{array}{c}\text { Mean Harris hip score } \\
\text { (uncemented) }\end{array}$ \\
\hline 1 month & 73.68 (Fair) & 71.28 (Fair) \\
\hline $3^{\text {rd }}$ month & 83.09 (Good) & 86.88 (Good) \\
\hline $6^{\text {th }}$ month & 91.75 (Excellent) & 93.17 (Excellent) \\
\hline $12^{\text {th }}$ month & 94.10 (Excellent) & 94.43 (Excellent) \\
\hline
\end{tabular}

Table 3: Comparison of Harris Hip Score at 12 months Post-op between Cemented and Uncemented Groups $(\mathrm{N}=50)$

\begin{tabular}{|c|c|c|}
\hline \multirow{2}{*}{ Harris Hip Score } & \multicolumn{2}{|c|}{ Group } \\
\cline { 2 - 3 } & $\begin{array}{c}\text { Cemented (n=25) } \\
\mathbf{n}(\mathbf{\%})\end{array}$ & $\begin{array}{c}\text { Uncemented (n=25) } \\
\mathbf{n}(\boldsymbol{\%})\end{array}$ \\
\hline $80-90$ (Good) & $6(28.57)$ & $7(30.43)$ \\
\hline $90-100$ (Excellent) & $15(71.43)$ & $16(69.57)$ \\
\hline Mean (SD) & $94.10(6.63)$ & $94.43(5.92)$ \\
\hline \multicolumn{2}{|c|}{ Unpaired t Test, P Value =0.862, Not Significant } \\
\hline
\end{tabular}

\section{Discussion}

With the trend of global aging, femoral neck fractures have become an increasingly serious problem for elderly patients. Hemiarthroplasty, as an effective treatment, can help resume the walking ability as soon as possible, thereby reducing the risk of respiratory infection and urinary tract infection. In elderly patients with a displaced femoral neck fracture hemiarthroplasty is therefore the widely accepted treatment of choice. Comparisons between cemented hemiarthroplasty and uncemented hemiarthroplasty have mostly favoured cemented fixation because of superior outcome in pain relief, better postoperative hip function recovery, less prosthesis loosening and periprosthetic fractures ${ }^{[2]}$. On the other hand, many hip fracture patients have significant cardiovascular and cerebral co-morbidities with little functional reserve. In these frail patients, operative time and blood loss can influence outcome. Therefore, some surgeons prefer to apply the uncemented hemiarthroplasty technique because they believe it can reduce operation time, and intraoperative blood loss. In addition, there is considerable evidence that cementing has potential physiologically adverse side effects.

In our study, the average age incidence of the patients was 75.02 years, with most patients $(68 \%)$ between $70-80$ years. It was comparable to various studies, mean age in the study of Wender Fifved ${ }^{[3]}$ et al was 70 years, mean age incidence in Li-Xing et al. ${ }^{[4]}$ study was 78 years.

Total number of females in the study were $27(54 \%)$ and number of male were $23(46 \%)$. There is a slightly higher incidence of female in the study. Female preponderance has been reported in several series Moore [5] 1957: 62.5\%, Campbell (1960): 80.9\%, Anderson \& Neilson [6] (1972): $85 \%$.

Majority of the patients $(84 \%)$ had trauma due to fall at home due to slip in the bathroom or while walking. This is in accordance with majority of the series reported - Ingalhalikar [7] (1987), Seth ${ }^{[8]}$ (1987), Scott and Gray (1980), Evarts [9] (1973)

In the cemented group the mean duration of surgery was 94.40 minutes (SD-12.18) with a mean amount of blood loss of 235.80 millilitres (SD-37.46), whereas in the uncemented group the mean duration of surgery was 60.80 minutes (SD11.42) and mean amount of blood loss being 183.20 millilitres (SD-29.11). Surgical time and blood loss was greater for the cemented cohort than the uncemented. The difference between the groups was significant $(\mathrm{P}<0.0001)$. Wender Figved MD et al. ${ }^{[3]}$ reported duration of 70.2 min with a blood loss of $300 \mathrm{ml}$ in uncemented group and 82.6 min with a blood loss of $390 \mathrm{ml}$ in the cemented group. Jaimo Ahn MD, $\mathrm{PhD}, \mathrm{Li}-X i n g$ Man MD et al. ${ }^{[4]}$ in their study recorded two operative parameters of blood loss and surgical time was lower for the uncemented cohorts. The weighted average blood loss was $476 \mathrm{~mL}$ for the cemented and $338 \mathrm{~mL}$ for the uncemented groups. Surgical time was greater for the cemented cohort than the uncemented (95 minutes versus 80 minutes, respectively).

All the cases were stable intraoperatively. There were no cases of intraoperative dislocation or peri prosthetic fracture. There were no conversion of uncemented bipolar hemiarthroplasty to cemented ones. R.J.K. Khan et al. ${ }^{[10]}$ reported three iatrogenic periprosthetic fractures, all occurring in the uncemented group. Foster et al. ${ }^{[11]}$ in 2005 from Northern Ireland (15). In their retrospective analysis of 244 patients of which 70 patients had uncemented prosthesis, $7 \%$ $(5 / 70)$ of patients with uncemented prosthesis suffered a periprosthetic fracture. Wender Figved MD et al. ${ }^{[3]}$ reported Intraoperative periprosthetic fracture one case $(0.9 \%)$ in cemented group \& $2(1.9 \%)$ in uncemented groups. There was no cases reported in our study studies have reported higher rates of intraoperative periprosthetic fracture in uncemented hemiarthroplasty.

There were $16 \%$ ( 4 cases) of cemented and $8 \%$ ( 2 cases) of uncemented cases were infection. The cemented group had slightly higher number of infection, although the $\mathrm{P}$ value is not less than 0.001, so it is not significant. Wender Figved MD et al. ${ }^{[3]}$ in his study reported only one case of superficial infection $(0.9 \%)$ in the cemented group.

There were no cases of dislocation in our study. Manoj Kumar Rajak et al. reported 3\% prosthesis dislocation following bipolar hemiarthroplasty ${ }^{[12]}$. Unwin et al. reported a $6.5 \%$ dislocation rate among all their patients, with those having posterior approaches being three times more likely to dislocate ${ }^{[13]}$.

There were total 4 cases $(16 \%)$ of mortality in cemented group and 2 cases (8\%) in uncemented group. No cases of intraoperative cardiac arrest or perioperative myocardial infarction leading to death which was seen in our study. D'Angelo et al. reported $25 \%$ one year follow up mortality rate following hemiarthroplasty ${ }^{[14]}$. Eiskjar reported $20 \%$ mortality rate at six months and $28 \%$ mortality rate at oneyear.

Though the cemented group had better functional outcome (although insignificant) in terms of Harris Hip Score at the first month, over the period at the end of one year the functional outcome of the two groups are similar.

One year Harris Hip Score results were equivalent and there were no differences in ability to walk, use of analgesics, or place of living in both the groups in the study reported by Wender Figved MD et al. ${ }^{[3]}$. Deangelis JP et al. ${ }^{[15]}$ concluded in the treatment of non-pathologic displaced femoral neck fractures, the use of cemented and uncemented femoral components is associated with similar functional outcome at 1 year. At 30-day, 60-day, and 1-year follow-ups, no clinically or statistically significant differences were found in mortality, disposition, need for assistance with ambulation. 
M. I. Parker, MD et al. ${ }^{[16]}$ in their study noted no statistically significant difference between the cemented and the uncemented groups with regard to mortality, implant- related complications, re-operations or post-operative medical complications.

According to Veldman et al. ${ }^{[17]}$ systematic review and metaanalysis to compare the outcomes of cemented and cement less hemiarthroplasty of the hip, in elderly patients with a fracture of the femoral neck, there was no statistically significant difference for any other outcome between the two methods of fixation. In hemiarthroplasty of the hip using current generation stems, cemented stems result in fewer implant-related complications and similar mortality compared with cement less stems.

\section{Conclusion}

Hip fractures in the elderly represent a major public health concern. Fracture neck of femur is a geriatric disease more so common in elderly females. Based on our results the advantages of using an uncemented hemiarthroplasty are also less intraoperative bleeding, shorter duration of surgery which was statistically significant. Complications like infection were distributed in both cemented and uncemented hemiarthroplasty groups and were statistically insignificant. The functional outcome at the end of one year is similar in both the groups.

Based on results of this prospective study it could be concluded, that both cemented and uncemented hemiarthroplasty are accepted modality of treatment in transcervical femur neck fractures in elderly age group, as there is no statistical difference in the functional_outcome (Harris Hip Score) at the end of one year .

\section{Acknowledgment}

Conflict of interest: Nil

Financial Support and Sponsorship: Nil

\section{References}

1. Crabtree N, Loveridge N, Parker M, Rushton N, Power J, Bell KL et al. Intracapsular hip fracture and the regionspecific loss of cortical bone: analysis by peripheral quantitative computed tomography. J Bone Miner Res 2001;16(7):1318-28. doi:10.1359/jbmr.2001.16.7.1318.

2. Parker MI, Pryor G, Gurusamy K. Cemented versus uncemented hemiarthroplasty for intracapsular hip fractures: A randomised controlled trial in 400 patients. J Bone Joint Surg Br. 2010;92(1):116-22.

3. Figved W, Opland V, Frihagen F, Jervidalo T, Madsen JE, Nordsletten L. Cemented versus uncemented hemiarthroplasty for displaced femoral neck fractures. Clin Orthop Relat Res 2009;467(9):2426-35.

4. Jaimo Ahn, Li-Xing Man, Sang Do Park, Jeffrey F Sodl. Systematic Review of Cemented and Uncemented Hemiarthroplasty Outcomes for Femoral Neck Fractures, Clin Orthop Relat Res 2008;466:2513-2518.

5. Austin T. Moore: The self locking metallic hip prosthesis. JBJS 1957;39A:811-27.

6. Ong BC, Maurer SG, Aharonoff GB, Zuckerman JD, Koval KJ. Unipolar versus bipolar hemiarthroplasty: functional outcome after femoral neck fracture at a minimum of thirty-six months of follow-up. J Orthop Trauma 2002;16(5):317-22.

7. Ingalhalikar V.T. Shekar Kumta. Fracture neck femur anatomical and biomechanical aspects. Clinc. Orthop.
India 1987.

8. Seth MK. (Col). Stress fractures of the neck of femur. Clinical Orthropaedics India 1987;1:105-109

9. Evarts CM. Endoprosthesis as the primary treatment of femoral neck fractures. Clinc. Orthop 1973;92:69-76.

10. Khan RJ, MacDowell A, Crossman P, Datta A, Jallali N, Arch BN et al. Cemented or uncemented hemiarthroplasty for displaced intracapsular femoral neck fractures. Int Orthop. 2002;26:229-232

11. Foster AP, Thompson NW, Wong J, Charlwood AP. Periprosthetic femoral fractures--a comparison between cemented and uncemented hemiarthroplasties. Injury. 2005;36(3):424-429

12. Rajak MK, Jha R, Kumar P, Thakur R. Bipolar hemiarthroplasty for intracapsular femoral neck fractures in elderly patients. J of orthop. Surg. (Hong Kong). 2013;21(3):313-6.

13. Unwin AJ, Thomas M. Dislocation after hemiarthroplasty of the hip: a comparison of the dislocation rate after posterior and lateral approaches to the hip. Ann. of the Roy. Coll. of Surg. of Engl 1994;76(5):327-9.

14. D’Angelo F, Giudici M, Molina M, Margaria G. Mortality rate after hip hemiarthroplasty: analysis of risk factors in 299 consecutives cases. J. of Orthop. and Traumatol 2005;6(3):111-6.

15. Deangelis JP, Ademi A, Staff I, Lewis CG. Cemented versus uncemented hemiarthroplasty for displaced femoral neck fractures: a prospective randomized trial with early follow-up. J Orthop Trauma 2012;26(3):13540.

16. Parker MJ, Gurusamy KS, Azegami S. Arthroplasties (with and without bone cement) for proximal femoral fractures in adults. Cochrane Database Syst Rev. 2010, CD001706.

17. Veldman, Hidde, Heyligers, Ide, Grimm, Bernd AEJ, Boymans T. Cemented versus cementless hemiarthroplasty for a displaced fracture of the femoral neck A Systematic Review and Meta-Analysis of Current Generation Hip Stems. The Bone \& Joint Journal 2017;99:421-431. 